\title{
ON MULTIPLICATORS IN HÖLDER SPACES WITH NONHOMOGENEOUS METRIC*
}

\section{O. LADYZHENSKAYA ${ }^{\dagger}$}

Abstract. We give a criterion when function $\hat{m}: E^{n} \rightarrow \mathbb{C}$ is a multiplicator in Hölder spaces with nonhomogeneous metrics. This criterion is applied to nonstationary system of hydrodynamical type.

I know two criterions when function $\hat{m}: E^{n} \rightarrow C$ is a multiplicator in the space $L^{\gamma}\left(E^{n}\right)$. The first of them goes back to Marcinkievicz (see [1] - [3]). It has found numerous applications to the study of elliptic equations and systems. But it is not applicable to parabolic equations. Even for $\hat{m}(\xi, \lambda)=\frac{\xi_{i} \xi_{k}}{i \lambda+\xi^{2}}$ arising for heat equation this criterion does not fulfilled. For this $\hat{m}$ the criterion given by Lizorkin and Stein ([4], [5]) can be used. As it was shown in [6] the later can be also applied to functions $\hat{m}$ which we have got solving the Cauchy problem for the system

$$
\begin{gathered}
\partial_{t} u_{j}-D_{j k, l m} \partial_{x_{k} x_{m}}^{2} u_{l}+\partial_{x_{j}} q=f_{j}, \quad j=1,2,3, \\
\operatorname{div} u=0
\end{gathered}
$$

with coefficients $D_{j k, l m}$ fixed at a point $(x, t)$. System (1) is a linearization (up to lower nonsignificant terms) of the Modified Navier-Stokes system (see [7], [8], etc)

$$
\partial_{t} v+v \cdot \nabla v-\left.\operatorname{div} \frac{\partial D(\varepsilon)}{\partial \varepsilon}\right|_{\varepsilon=\varepsilon(v)}+\nabla p=g, \quad \operatorname{div} v=0
$$

where $D: M_{\text {sym }}^{3 \times 3} \rightarrow E_{+}^{1}=[0, \infty)$ is a smooth convex function of the symmetric tensors $\varepsilon=\left(\varepsilon_{i j}\right)$ and $\varepsilon(v)=\frac{1}{2} \nabla v+\frac{1}{2}(\nabla v)^{T}$ (here $\nabla v$ is the gradient of $v$ with respect to the space variables $\left.x=\left(x_{1}, x_{2}, x_{3}\right) \in E^{3}\right)$.

In (2) velocity field $v$ and pressure $p$ are unknown functions and in (1) their variations $u$ and $q$ are the functions under consideration. At first, we reduce the nonhomogeneous initial data $\left.v\right|_{t=0}=v^{0}$ to the data

$$
\left.v\right|_{t=0}=0
$$

and study the Cauchy problem (1), (3), supposing that $f(x, 0) \equiv 0$. After that we put $u, q$ and $f$ equal to zero for $(x, t) \in E^{3} \times E_{-}^{1}=(-\infty, 0)$ and pass to their Fourier images $\hat{u}, \hat{q}$ and $\hat{f}$. For them we get the system

$$
i \lambda \hat{u}_{j}+A_{j l}(\xi) \hat{u}_{l}+i \xi_{j} \hat{q}=\hat{f}_{j}, \quad j=1,2,3, \quad \xi_{k} \hat{u}_{k}=0,
$$

where $A_{j l}(\xi)=D_{j k, l m} \xi_{k} \xi_{m}$. The variables $\xi=\left(\xi_{1}, \xi_{2}, \xi_{3}\right)$ are dual to variables $\left(x_{1}, x_{2}, x_{3}\right)$ and $\lambda$ is dual to $t$. The conditions which are usually put on $D$ (see [7] - [9], etc) generate for $D_{j k, l m}$ the restrictions

$$
\nu_{0}|\varkappa|^{2} \leq D_{j k, l m} \varkappa_{j k} \varkappa_{l m} \leq \mu_{0}|\varkappa|^{2}
$$

* Received December 25, 1999.

† St.-Petersburg Branch of Steklov Mathematical Institute, Russian Academy of Science, Fontanka 27, St.-Petersburg, 191011, Russia (ladyzhen@pdmi.ras.ru). The work is partly supported by RFFI no. 99-01-00108 and by INTAS no. 96-0835. 
where $\nu_{0}$ and $\mu_{0}$ are positive constants, $\varkappa$ is arbitrary symmetric tensor and $|\varkappa|^{2}=$ $\sum_{j, k} \varkappa_{j k} \varkappa_{j k}$.

The relations

$$
D_{j k, l m}=D_{k j, l m}=D_{k j, m l}=D_{j k, m l}=D_{l m, j k}
$$

are also fulfilled. According to (5) and (6) matrixes $A(\xi)=\left(A_{j l}(\xi)\right)$ are symmetric and they satisfy the inequalities

$$
A_{j l}(\xi) \eta_{j} \eta_{l} \geq \nu_{0}|\xi|^{2}|\eta|^{2}
$$

for arbitrary vectors $\xi, \eta \in E^{3}$.

System (4) is uniquely solvable for all real $\xi$ and $\lambda$ except the axis $\lambda$ (i.e. when $\xi=0$ and $\lambda$ is arbitrary). The principal matrix $B(\xi, \lambda)$ of the system (4) satisfies the inequalities

$$
|\operatorname{det} B(\xi, \lambda)| \geq \nu_{1}|\xi|^{2}\left(|\xi|^{4}+|\lambda|^{2}\right)
$$

with a positive constant $\nu_{1}$ and arbitrary $(\xi, \lambda) \in E^{4}$. The solution $\hat{u}, \hat{q}$ of (4) has the form

$$
\begin{gathered}
\hat{u}_{j}(\xi, \lambda)=\frac{1}{\operatorname{det} B(\xi, \lambda)} \sum_{k=1}^{3} C_{j k}(\xi, \lambda) \hat{f}_{k}(\xi, \lambda), \quad j=1,2,3 \\
\hat{q}(\xi, \lambda)=\frac{1}{\operatorname{det} B(\xi, \lambda)} \sum_{k=1}^{3} C_{4 k}(\xi, \lambda) \hat{f}_{k}(\xi, \lambda) .
\end{gathered}
$$

The functions $C_{j k}, j<4$ have the structure

$$
C_{j k}(\xi, \lambda)=C_{j k, l m}(\xi, \lambda) \xi_{l} \xi_{m}
$$

where $C_{j k, l m}$ are 2-homogeneous polynomials in the following sense:

$$
C_{j k, l m}\left(a \xi, a^{2} \lambda\right)=a^{2} C_{j k, l m}(\xi, \lambda)
$$

Functions $C_{4 k}$ are 5-homogeneous polynomials, i.e.

$$
C_{4 k}\left(a \xi, a^{2} \lambda\right)=a^{5} C_{4 k}(\xi, \lambda) .
$$

For $f$ satisfying the condition

$$
\operatorname{div} f=0
$$

functions $\hat{f}=F(f)$ satisfy the relations

$$
\xi_{k} \hat{f}_{k}(\xi, \lambda)=0
$$

and $C_{4 k}$ have the following structure

$$
C_{4 k}(\xi, \lambda)=C_{4 k, l m n}(\xi, \lambda) \xi_{l} \xi_{m} \xi_{n}
$$

with 2-homogeneous polynomials $C_{4 k, l m n}$. Besides (7), function $\operatorname{det} B$ has the properties

$$
\operatorname{det} B(\xi, \lambda)=b_{k l}(\xi, \lambda) \xi_{k} \xi_{l}
$$


and

$$
b_{k l}\left(a \xi, a^{2} \lambda\right)=a^{4} b_{k l}(\xi, \lambda) .
$$

It is not difficult to calculate that the fractions

$$
\frac{C_{j k}(\xi, \lambda) \xi_{l} \xi_{m}}{\operatorname{det} B(\xi, \lambda)}, \quad j, k, l, m=1,2,3
$$

and also the fractions

$$
\frac{C_{4 k}(\xi, \lambda) \xi_{l}}{\operatorname{det} B(\xi, \lambda)}, \quad k, l=1,2,3,
$$

(when $C_{4 k}$ have the form $\left(9_{3}\right)$ ) satisfy the conditions of Theorem 2 which we formulate and prove below. Thus, they are multiplicators in the Holder space $H^{(\gamma, \gamma / 2)}\left(Q_{T}\right)$ with parabolic metric

$$
\rho\left((x, t),\left(x^{\prime}, t^{\prime}\right)\right)=\left|x-x^{\prime}\right|+\left|t-t^{\prime}\right|^{1 / 2}
$$

and any $\gamma \in(0,1)$. The norm in $H^{(\gamma, \gamma / 2)}\left(Q_{T}\right), Q_{T}=E^{3} \times(0, T)$, is determined by the equality

$$
|u|_{H^{(\gamma, \gamma / 2)}}\left(Q_{T}\right)=\sup _{Q_{T}}|u|+\langle u\rangle_{Q_{T}}^{(\gamma, \gamma / 2)}
$$

where the second term is called Hölder constant and has the form

$$
\begin{gathered}
\langle u\rangle_{Q_{T}}^{(\gamma, \gamma / 2)}=\sup _{(x, t),\left(x^{\prime}, t^{\prime}\right) \in Q_{T}} \frac{\left|u(x, t)-u\left(x^{\prime}, t^{\prime}\right)\right|}{\rho^{\gamma}\left((x, t),\left(x^{\prime}, t^{\prime}\right)\right)} \\
\rho^{\gamma}\left((x, t),\left(x^{\prime}, t^{\prime}\right)\right) \leq 1
\end{gathered}
$$

So, with the help of Theorem 2 we get the statement

Theorem 1. For solutions $u$, $q$ of the Cauchy problem (1), (3) with $f \in L^{2}\left(Q_{T}\right) \cap$ $H^{(\gamma, \gamma / 2)}\left(Q_{T}\right)$ the following estimates

$$
\left\|\partial_{t} u, \partial_{x x}^{2} u, \partial_{x} u, u\right\|_{L^{2}\left(Q_{T}\right)} \leq C\|f\|_{L^{2}\left(Q_{T}\right)}
$$

and

$$
\left\langle\partial_{x x}^{2} u\right\rangle_{Q_{T}}^{(\gamma, \gamma / 2)} \leq C(\gamma)\langle f\rangle_{Q_{T}}^{(\gamma, \gamma / 2)}
$$

hold. If, additionally, $f$ satisfy $\left(9_{1}\right)$ and $f(x, 0)=0$, then

$$
\langle\nabla q\rangle_{Q_{T}}^{(\gamma, \gamma / 2)} \leq C(\gamma)\langle f\rangle_{Q_{T}}^{(\gamma, \gamma / 2)}
$$

is also true.

Basing on this theorem the theorem on unique solvability of the Cauchy problem for system (1) in space $W_{2}^{2,1}\left(Q_{T}\right) \cap H^{2+\gamma, 1+\gamma / 2}\left(Q_{T}\right)$ for $u$ and space $L^{2}\left(Q_{T}\right) \cap$ $H^{\gamma, \gamma / 2}\left(Q_{T}\right)$ for $\nabla q$ is proved by known methods. Let us point out that in the work [6] of G. Seregin and myself the estimate $\left(12_{1}\right)$ in the space $L^{\gamma}\left(Q_{T}\right)$ with arbitrary $\gamma \in(1, \infty)$ is proved. To do this we have convinced that the functions $\xi^{2}(\operatorname{det} B(\xi, \lambda))^{-1} C_{j k}(\xi, \lambda), j, k=1,2,3$, satisfy Lizorkin-Stein criterion and hence they are multiplicators in the spaces $L^{\gamma}\left(E^{4}\right), \gamma \in(1, \infty)$. In [6] we have proved also the estimate $\left(12_{2}\right)$ (but not $\left(12_{3}\right)$ ). We did this by special technique developed for the study of parabolic systems (see Campanato [10] and others). Here inequalities $\left(12_{k}\right)$, $k=2,3$, are proved with the help of following criterion. 
Theorem 2. Let $\hat{m}$ be a bounded function of variables $\xi=\left(\xi^{\prime}, \xi_{n}\right) \in E^{n}, \xi^{\prime}=$ $\left(\xi_{1}, \ldots, \xi_{n-1}\right)$ satisfying inequalities

$$
\left\|\check{m}_{j}\right\|_{W_{2}^{s}(\omega)} \leq \mu, \quad \forall j \in \mathbb{Z}=\{\ldots,-1,0,1, \ldots\},
$$

with $a \mu<\infty$ and $s>n / 2$. In (13) $\check{m}_{j}(\xi)=\hat{m}\left(2^{j} \xi^{\prime}, 2^{j / \alpha} \xi_{n}\right)$ with $\alpha \in(0,1]$ and $\omega=\left\{\xi \quad \mid \frac{1}{4} \leq \rho(\xi) \leq 4\right\}$ where $\rho(\xi)=\left|\xi^{\prime}\right|+\left|\xi_{n}\right|^{\alpha}$ and $\left|\xi^{\prime}\right|=\sum_{k=1}^{n-1}\left|\xi_{k}\right|$. Such $\hat{m}$ is $a$ multiplicator in Hölder spaces $H^{(\gamma, \alpha \gamma)}\left(E^{n}\right), \gamma \in(0,1)$, i.e.

$$
\langle m * u\rangle_{E^{n}}^{(\gamma, \alpha \gamma)} \leq C(\gamma, \alpha, n) \mu\langle u\rangle_{E^{n}}^{(\gamma, \alpha \gamma)}
$$

where $m=F^{-1}(\hat{m})$ and $u$ is an arbitrary function from $C_{0}^{1}\left(E^{n}\right)$.

Here we have used the notations

$$
\begin{gathered}
\langle u\rangle_{E^{n}}^{(\gamma, \alpha \gamma)}=\sup _{\left(x^{\prime}, x_{n}\right),\left(y^{\prime}, y_{n}\right) \in E^{n}} \frac{\left|u\left(x^{\prime}, x_{n}\right)-u\left(y^{\prime}, y_{n}\right)\right|}{\rho^{\gamma}(x-y)} \\
\rho(x-y) \leq 1
\end{gathered}
$$

and $m=F^{-1}(\widehat{m})$ is the inverse Fourier image of $\widehat{m}$.

Above we have used this theorem for $\alpha=1 / 2$ and 0 -homogeneous functions, so that for them $\check{m}_{j}(\xi)=\widehat{m}(\xi)$ and we have examine (13) only for function $\widehat{m}(\xi)$ itself.

We proof Theorem 2 following to the same plan that was applied by Hörmander for the proof of Theorem 7.9.6 from [3], but we work with other auxiliary functions. Thus, we take a smooth function $\psi: E_{+}^{1} \rightarrow[0,1]$ which is equal 1 on the interval $[0,1]$ and zero on $[2, \infty)$ and the function $\varphi: E_{+}^{1} \rightarrow[0,1]$ determining by equality $\varphi(\rho)=\psi(\rho)-\psi(2 \rho), \rho \in E_{+}^{1}$. The later is equal zero on the sets $\left[0, \frac{1}{2}\right]$ and $[2, \infty)$. Let us introduce also the functions $\varphi_{j}: E_{+}^{1} \rightarrow[0,1]$ by equalities $\varphi_{j}(\rho)=\varphi\left(\frac{\rho}{2^{j}}\right)$. It is easy to see that

$$
\sum_{j=-\infty}^{\infty} \varphi_{j}(\rho)=1, \quad \rho \in(0, \infty) .
$$

Now, using $\varphi$ and $\varphi_{j}$, we determine the functions $\widehat{\phi}$ and $\widehat{\phi}_{j}: E^{n} \rightarrow[0,1]$ in the following way:

$$
\begin{gathered}
\widehat{\phi}=\varphi \circ \rho, \quad \text { i.e. } \widehat{\phi}(\xi)=\varphi(\rho(\xi)) \\
\widehat{\phi}_{j}=\varphi_{j} \circ \rho, \quad \text { i.e. } \widehat{\phi}_{j}(\xi)=\varphi_{j}(\rho(\xi))= \\
\varphi\left(\frac{\rho(\xi)}{2^{j}}\right)=\varphi\left(\rho\left(\frac{\xi^{\prime}}{2^{j}}, \frac{\xi_{n}}{2^{j / \alpha}}\right)\right)=\widehat{\phi}\left(\frac{\xi^{\prime}}{2^{j}}, \frac{\xi_{n}}{2^{j / \alpha}}\right) .
\end{gathered}
$$

The equality $\left(16_{1}\right)$ generate the following

$$
\sum_{j=-\infty}^{\infty} \widehat{\phi}_{j}(\xi)=1, \quad \xi \in E^{n} \backslash\{0\} .
$$

According to $\left(16_{k}\right), k=1, \ldots, 4$ we represent $\widehat{u}(\xi)=F(u)(\xi)$ as the sum $\widehat{u}(\xi)=$ $\sum_{j} \widehat{u}_{j}(\xi)$ where $\widehat{u}_{j}(\xi)=\widehat{u}(\xi) \widehat{\phi}_{j}(\xi)$. Let $\phi_{j}$ be the inverse Fourier transform of $\widehat{\phi}_{j}$, i.e. 
$\phi_{j}=F^{-1}\left(\widehat{\phi}_{j}\right)$. Then using a change of variables we get

$$
\begin{gathered}
\phi_{j}(x)=F^{-1}\left(\widehat{\phi}_{j}\right)(x)=\int e^{i x \xi} \widehat{\phi}\left(\frac{\xi^{\prime}}{2^{j}}, \frac{\xi_{n}}{2^{j / \alpha}}\right) d \xi \\
=2^{(n-1) j+j / \alpha} \int e^{i 2^{j} x^{\prime} \eta^{\prime}+i 2^{j / \alpha} x_{n} \eta_{n}} \widehat{\phi}\left(\eta^{\prime}, \eta_{n}\right) d \eta=2^{(n-1) j+j / \alpha} \phi\left(2^{j} x^{\prime}, 2^{j / \alpha} x_{n}\right)
\end{gathered}
$$

where $\phi(x)=F^{-1}(\widehat{\phi})(x)=\int e^{i x \xi} \widehat{\phi}(\xi) d \xi$

Let us introduce a smooth function $\theta: E_{+}^{1} \rightarrow[0,1]$, which is equal to 1 for $\rho \in\left[\frac{1}{2}, 2\right]$, to zero for $\rho \in\left[0, \frac{1}{4}\right]$ and $\rho \geq 4$, and the function $\chi: E^{n} \rightarrow[0,1]$ determined by equalities $\chi(\xi)=\theta(\rho(\xi)), \xi \in E^{n}$. It is clear that $\widehat{\phi}(\xi)=\widehat{\phi}(\xi) \chi(\xi)$ and $\widehat{\phi}_{j}(\xi)=$ $\widehat{\phi}_{j}(\xi) \chi\left(\frac{\xi^{\prime}}{2^{j}}, \frac{\xi_{n}}{2^{j / \alpha}}\right)$ and therefore

$$
\widehat{u}_{j}(\xi)=\widehat{u}(\xi) \widehat{\phi}_{j}(\xi)=\widehat{u}(\xi) \widehat{\phi}\left(\frac{\xi^{\prime}}{2^{j}}, \frac{\xi_{n}}{2^{j / \alpha}}\right) \chi\left(\frac{\xi^{\prime}}{2^{j}}, \frac{\xi_{n}}{2^{j / \alpha}}\right)=\widehat{u}_{j}(\xi) \chi\left(\frac{\xi^{\prime}}{2^{j}}, \frac{\xi_{n}}{2^{j / \alpha}}\right) .
$$

Let

$$
v \equiv m * u \text {. }
$$

Then for its Fourier image $\widehat{v}$ we have

$$
\widehat{v}(\xi)=\widehat{m}(\xi) \widehat{u}(\xi)=\sum_{j} \widehat{m}(\xi) \widehat{u}_{j}(\xi)=\sum_{j} \widehat{v}_{j}(\xi)
$$

where $\widehat{v}_{j}(\xi)=\widehat{m}_{j}(\xi) \widehat{u}_{j}(\xi)$ and $\widehat{m}_{j}(\xi)=\widehat{m}(\xi) \chi\left(\frac{\xi^{\prime}}{2^{j}}, \frac{\xi_{n}}{2^{j / \alpha}}\right)$

Let us show that $F^{-1}\left(\widehat{m}_{j}\right)$ have the uniformly (with respect to $\mathrm{j}$ ) bounded norms in $L^{1}$. For this purpose we remark that

$$
\begin{aligned}
& \left\|F^{-1} \widehat{m}_{j}\right\|_{L^{1}\left(E^{n}\right)}=\int_{E^{n}} d x\left|\int e^{i x \xi} \widehat{m}(\xi) \chi\left(\frac{\xi^{\prime}}{2^{j}}, \frac{\xi_{n}}{2^{j / \alpha}}\right) d \xi\right| \\
& =\int d y\left|\int e^{i \eta y} \widehat{m}\left(2^{j} \eta^{\prime}, 2^{j / \alpha} \eta_{n}\right) \chi(\eta) d \eta\right|=\left\|F^{-1} M_{j}\right\|_{L^{1}}
\end{aligned}
$$

where $M_{j}(\eta)=\widehat{m}\left(2^{j} \eta^{\prime}, 2^{j / \alpha} \eta_{n}\right) \chi(\eta)=\check{m}_{j}(\eta) \chi(\eta)$.

In virtue of well known inequalities, for any $M_{j}$

$$
\left\|F^{-1} M_{j}\right\|_{L^{1}\left(E^{n}\right)} \leq C(n, s)\left\|M_{j}\right\|_{W_{2}^{s}\left(E^{n}\right)}, \quad s>\frac{n}{2} .
$$

The right hand side of (19) is uniformly bounded due to our condition (13) and our choice of $\chi$. Thus we have

$$
\left\|F^{-1} \hat{m}_{j}\right\|_{L^{1}} \leq c_{0} \mu \quad \text { for any } j \in \mathbb{Z}
$$

Due to (17) we have

$$
\begin{gathered}
u_{j}(x)=F^{-1}\left(\hat{u}_{j}\right)=F^{-1}\left(\hat{u} \hat{\phi}_{j}\right) \\
=u * F^{-1}\left(\hat{\phi}_{j}\right)=u * \phi_{j}=2^{(n-1) j+j / \alpha} \int u(x-y) \phi\left(2^{j} y^{\prime}, 2^{j / \alpha} y_{n}\right) d y^{\prime} d y_{n} \\
=\int\left(u\left(x^{\prime}-\frac{z^{\prime}}{2^{j}}, x_{n}-\frac{z_{n}}{2^{j / \alpha}}\right)-u(x)\right) \phi(z) d z
\end{gathered}
$$


Here we have used $\hat{\phi}(0)=0=\int \phi(z) d z$. Analogously we get

$$
\partial_{x_{k}} u_{j}(x)=2^{j} \int\left(u\left(x^{\prime}-\frac{z^{\prime}}{2^{j}}, x_{n}-\frac{z_{n}}{2^{j / \alpha}}\right)-u(x)\right) \partial_{z_{k}} \phi(z) d z, \quad k<n
$$

and

$$
\partial_{x_{n}} u_{j}(x)=2^{j / \alpha} \int\left(u\left(x^{\prime}-\frac{z^{\prime}}{2^{j}}, x_{n}-\frac{z_{n}}{2^{j / \alpha}}\right)-u(x)\right) \partial_{z_{n}} \phi(z) d z .
$$

The following inequalities we deduce from $\left(21_{k}\right)$ :

$$
\begin{aligned}
& \sup _{x \in E^{n}}\left|u_{j}(x)\right| \leq\langle u\rangle^{(\gamma, \alpha \gamma)} \int\left(\frac{\left|z^{\prime}\right|}{2^{j}}+\frac{\left|z_{n}\right|^{\alpha}}{2^{j}}\right)^{\gamma} \phi(z) d z \\
& \equiv c_{1}(\gamma, \alpha) 2^{-j \gamma}\langle u\rangle^{(\gamma, \alpha \gamma)}, \\
& \sup _{x \in E^{n}}\left|\partial_{x_{k}} u_{j}(x)\right| \leq\langle u\rangle^{(\gamma, \alpha \gamma)} 2^{j-j \gamma} \int\left(\left|z^{\prime}\right|+\left|z_{n}\right|^{\alpha}\right)^{\gamma}\left|\partial_{z_{k}} \phi(z)\right| d z \\
& \equiv c_{2}(\gamma, \alpha) 2^{j-j \gamma}\langle u\rangle^{(\gamma, \alpha \gamma)},
\end{aligned}
$$

for $k<n$ and

$$
\begin{gathered}
\sup _{x \in E^{n}}\left|\partial_{x_{n}} u_{j}(x)\right| \leq\langle u\rangle^{(\gamma, \alpha \gamma)} 2^{j / \alpha-j \gamma} \int\left(\left|z^{\prime}\right|+\left|z_{n}\right|^{\alpha}\right)^{\gamma}\left|\partial_{z_{n}} \phi(z)\right| d z \\
\equiv c_{3}(\gamma, \alpha) 2^{j / \alpha-j \gamma}\langle u\rangle^{(\gamma, \alpha \gamma)}
\end{gathered}
$$

These inequalities and estimate (20) we use to evaluate functions $v_{j}$ :

$$
v_{j}(x) \equiv F^{-1}\left(\hat{v}_{j}\right)=F^{-1}\left(\hat{m}_{j} \hat{u}_{j}\right)=m_{j} * u_{j}=\int u_{j}(x-y) m_{j}(y) d y
$$

where $m_{j}=F^{-1}\left(\hat{m}_{j}\right)$ and $\hat{m}_{j}$ is determined after $\left(18_{2}\right)$. Namely

$$
\sup _{x}\left|v_{j}(x)\right| \leq\left\|m_{j}\right\|_{L^{1}} \sup _{x}\left|u_{j}(x)\right| \leq c_{0} \mu c_{1}\langle u\rangle^{(\gamma, \alpha \gamma)} 2^{-j \gamma} \equiv d_{1} 2^{-j \gamma},
$$

$$
\sup _{x}\left|\partial_{x_{k}} v_{j}(x)\right| \leq\left\|m_{j}\right\|_{L^{1}} \sup _{x}\left|\partial_{x_{k}} u_{j}(x)\right| \leq c_{0} \mu c_{2}\langle u\rangle^{(\gamma, \alpha \gamma)} 2^{j-j \gamma} \equiv d_{2} 2^{j-j \gamma}
$$

$\left(23_{4}\right) \sup _{x}\left|\partial_{x_{n}} v_{j}(x)\right| \leq\left\|m_{j}\right\|_{L^{1}} \sup _{x}\left|\partial_{x_{n}} u_{j}(x)\right| \leq c_{0} \mu c_{3}\langle u\rangle^{(\gamma, \alpha \gamma)} 2^{j / \alpha-j \gamma} \equiv d_{3} 2^{j / \alpha-j \gamma}$,

Now we estimate $|v(x)-v(y)|$ for all $x, y$ from $E^{n}$ for which $\rho(x-y)=\left|x^{\prime}-y^{\prime}\right|+$ $\left|x_{n}-y_{n}\right|^{\alpha} \leq 1$, in the following way:

$$
|v(x)-v(y)| \leq \sum_{j}\left|v_{j}(x)-v_{j}(y)\right| \leq \sum_{j \geq n_{0}} \ldots+\sum_{j \leq n_{0}} \ldots \equiv S_{1}+S_{2},
$$

where $n_{0}=-\log _{2} \rho(x-y)$. For evaluation of $S_{1}$ we use $\left(23_{2}\right)$

$$
\begin{gathered}
S_{1} \leq \sum_{j \geq n_{0}} 2 \sup _{x}\left|v_{j}\right| \leq 2 d_{1} \sum_{j \geq n_{0}} 2^{-j \gamma} \\
=2 d_{1} 2^{-n_{0} \gamma} \sum_{k=0}^{\infty} \frac{1}{2^{\gamma k}} \equiv e_{1}(\gamma, \alpha) 2^{-n_{0} \gamma}=e_{1} \rho^{\gamma}(x-y) .
\end{gathered}
$$


For evaluation of $S_{2}$ we use $\left(23_{3}\right)$ and $\left(23_{4}\right)$ :

$$
\begin{gathered}
S_{2} \leq \sum_{j \leq n_{0}}\left(\sum_{k=1}^{n-1}\left|x_{k}-y_{k}\right| \sup _{x}\left|\partial_{x_{k}} v_{j}(x)\right|+\left|x_{n}-y_{n}\right| \sup _{x}\left|\partial_{x_{n}} v_{j}(x)\right|\right) \\
\leq \sum_{j \leq n_{0}}\left(d_{2}\left|x^{\prime}-y^{\prime}\right| 2^{j-j \gamma}+d_{3}\left|x_{n}-y_{n}\right| 2^{j / \alpha-j \gamma}\right) \\
\leq d_{2}\left|x^{\prime}-y^{\prime}\right| 2^{(1-\gamma) n_{0}} \sum_{k=0}^{\infty} \frac{1}{2^{k(1-\gamma)}}+d_{3}\left|x_{n}-y_{n}\right| 2^{n_{0}(1 / \alpha-\gamma)} \sum_{k=0}^{\infty} \frac{1}{2^{k(1 / \alpha-\gamma)}} \\
=e_{2}\left|x^{\prime}-y^{\prime}\right| \rho^{\gamma-1}(x-y)+e_{3}\left|x_{n}-y_{n}\right| \rho^{\gamma-1 / \alpha}(x-y) \leq\left(e_{2}+e_{3}\right) \rho^{\gamma}(x-y)
\end{gathered}
$$

Thus we have convinced that

$$
|v(x)-v(y)| \leq\left(e_{1}+e_{2}+e_{3}\right) \rho^{\gamma}(x-y) .
$$

So Theorem 2 is proved.

Remark. Theorem 2 is also true if we choose

$$
\rho(\xi)=\sum_{k=1}^{n}\left|\xi_{k}\right|^{\alpha_{k}}, \quad \alpha_{1}=1, \quad \alpha_{k} \in(0,1]
$$

Remark. In Theorem 2 instead of the condition (13) we can take the condition

$$
\left\|F^{-1} M_{j}\right\|_{L^{1}\left(E^{n}\right)} \leq \mu_{1}, \quad \text { for all } j \in \mathbb{Z}
$$

where $M_{j}$ are determined just before the inequality (19). In fact, as it is seen from the proof of Theorem 2, the condition (13) was used only for to get the estimate $\left(^{*}\right)$ (See (19) and (20)).

\section{REFERENCES}

[1] J. Marcinkievicz, Sur les multiplicators des series Fourier, Studia math., 8 (1939), pp. 78-91.

[2] S. G. Michlin, Multidimensional Singular Integrals and Integral Equations, GIFML, Moskow, 1962.

[3] L. Hörmander, The Analysis of Linear Partial Differential Operators I, Springer, 1983.

[4] P. I. Lizorkin, $\left(L_{p}, L_{q}\right)$-multiplicators of the Fourier integrals, Trudy MIAN, 89 (1967), pp. 231-248.

[5] E. Stein, Singular Integrals and Differentiability Properties of Functions, Princeton, New Jersey, 1970 .

[6] O. A. Ladyzhenskaya and G. A. Seregin, Coersive estimates for solutions to linearization of the Modifyed Navier-Stokes equations, Doklady of Russian Academy of Science, 370 (2000), pp. $738-740$.

[7] O. A. Ladyzhenskaya, On some nonlinear problems in the theory of continuous media, Proceedings of International Mathem. Congress (Moskow, 1966) (1968), Mir, Moskow, pp. 560573.

[8] O. A. LadyzhensKaya, Attractors for the modifications of the three-dimensional Navier-Stokes equations, Philos. Trans. Royal Soc. of London, Ser. A, 346 (1994), pp. 173-190.

[9] O. A. Ladyzhenskaya and G. A. Seregin, On smoothness of solutions to systems describing flows of generalized Newtonian fluids and estimates of the dimension of their attractors, Izvestiya of Russian Academy of Sciences, Ser. Mathem., 62:1 (1998), pp. 59-122. 
[10] S. Campanato, $L^{p}$-regularity and partial Hölder continuity for solutions of second order parabolic systems with strictly controlled growth, Ann. Math. Pure e Appl., 78 (1980), pp. 287310 . 